\title{
EFECTOS EN LA EJECUCIÓN DURANTE UNA TAREA DE IGUALACIÓN A LA MUESTRA SEGÚN EL TIPO Y EL ORDEN DE EXPOSICIÓN A LAS PRUEBAS DE TRANSFERENCIA ${ }^{1}$
}

\author{
Camilo Hurtado-Parrado*, Miguel Ángel Robayo, \\ Telmo Eduardo Peña-Correal \\ Universidad Nacional de Colombia
}

Recibido: marzo 17 de 2007

Revisado: mayo 18 de 2007

Aceptado: junio 27 de 2007

\section{Effects on the Performance during a Matching-To-sample Task due to THE TyPe and Order of Exposition to the Transference Tests}

\begin{abstract}
This study evaluated the effects of manipulating the type and order of presentation of transference tests. Twenty eight undergraduate students divided in 4 groups were exposed to a second order matching to sample procedure. The conditions of exposition were: ascending difficulty/complexity order of the tests, descending order and two randomly assigned orders. Results are discussed in terms of percentages of effectiveness; additionally, the latency is proposed as an alternative measure sensitive to the level of difficulty of this kind of tasks. Findings showed heterogeneity in the velocity of acquisition of the conditional discriminations during the training phase, even though the conditions of the task were equal for all the subjects. The exposition to the ascending and descending order seemed to affect negatively the effective behavioral adjustment, whereas one of the randomly assigned sequences seemed to be the best condition. The order of exposition to transference tests, in interaction with a history of early acquisition in the training phase, served to understand the findings of this study and to discuss the necessity of a systematical evaluation of the factors

\footnotetext{
El experimento reportado en este artículo constituyó el trabajo de grado de los dos primeros autores, integrantes del Grupo de Investigación en Conducta Simbólica del Departamento de Psicología de la Universidad Nacional de Colombia (Bogotá), dirigido por Telmo Eduardo Peña-Correal, Ph. D. (Marzo de 2004). Colombia.
}

* E-mail: hchurtadop@unal.edu.co / Dirección postal: Ciudad Universitaria Av. Cra. 30 No. 45-03 - Edificio 212, Bogotá, D.C.,
\end{abstract}


implied in the transference tests. It is suggested to assess the validity of different kind of transference tests and the convenience of some of them to be use in the investigation of the phenomena related to the effective and variable behavior.

Key words: transference, matching to simple procedure, geometric dimension, semantic dimension, intrasituational extrasituational and transituational behavior, extrainstance, extramodal, extrarelational and extradimensional transference, transference tests, conditional discriminations.

\section{RESUMEN}

Se evaluó el efecto de manipular el tipo y el orden de presentación de pruebas de transferencia intramodal, extramodal, extrarrelacional y extradimensional, después de un entrenamiento en relaciones de discriminación condicional. Se entrenó a 28 estudiantes universitarios en un procedimiento de igualación a la muestra de segundo orden con retroalimentación continua. Luego los participantes fueron divididos en cuatro grupos experimentales que variaron en el orden de presentación de las pruebas de transferencia. Las condiciones de exposición fueron: orden de complejidad/ dificultad ascendente, descendente, y dos grupos en donde el orden de las pruebas fue establecido aleatoriamente. Se analizaron los porcentajes de efectividad y una medida poco explorada en este tipo de estudios, el tiempo de latencia de respuesta, que fue propuesto como una variable sensible a la dificultad de una tarea de igualación. Se evidenció heterogeneidad en la velocidad de adquisición de las discriminaciones en la fase de entrenamiento, a pesar de que todos los sujetos fueron expuestos a la misma tarea. La exposición a las secuencias de prueba ascendente y descendente no favoreció el ajuste efectivo del comportamiento, mientras que la exposición a una de las secuencias asignadas de forma aleatoria pareció ser la que favoreció desempeños más altos y homogéneos. El orden en que se expusieron los participantes a los diferentes tipos de prueba de transferencia, en interacción con la historia de adquisición temprana durante el entrenamiento, dan cuenta de los resultados del estudio. Se discute la necesidad de abordar sistemáticamente el estudio de los factores implicados en las pruebas de transferencia.

Palabras clave: transferencia, igualación a la muestra, dimensión geométrica, dimensión semántica, comportamiento intrasituacional, extrasituacional y transituacional, transferencia extrainstancia, extramodal, extrarrelacional y extradimensional, pruebas de transferencia, discriminación condicional.

Cuando el comportamiento exhibido en una prueba es similar al mostrado en el entrenamiento, en términos de ajustarse a un criterio de efectividad, se dice que ha ocurrido transferencia de lo aprendido (Martínez, 2001). Comportarse efectivamente en una situación novedosa "b" (prueba), dependiendo de una historia previa en "a" (entrenamiento), conduce a preguntarse acerca de cuáles pueden ser y cómo se relacionan los factores que intervienen para que el comportamiento de los individuos se dé como si estuvieran frente a una situación en la cual ya habían sido exitosos.

Si se emplea como criterio la cantidad o cualidad de las características que comparte un conjunto de situaciones con las circunstancias iniciales donde tuvo lugar lo aprendido, es posible organizar dichas situaciones desde aquellas que son familiares, hasta otras más bien disímiles. Bajo esta presunción, y para propósitos experimentales, podemos denominar a las situaciones que comparten características con las de aprendizaje como condiciones de prueba.
Precisamente ésta es parte de la lógica subyacente a la metodología utilizada en un programa de investigación cuyo interés ha sido identificar los factores involucrados en la adquisición y transferencia del comportamiento inteligente, entendiendo éste como comportamiento variado y efectivo (Ribes, 1990; Varela \& Quintana, 1995; Varela \& Ribes, 2002). En dicha tradición de investigación, la variabilidad se ha estudiado observando cómo la conducta de los individuos se ajusta ante diversas situaciones novedosas, de la misma manera en que lo había hecho en una situación inicial de aprendizaje; en otras palabras, al observar fenómenos de transferencia del comportamiento. La efectividad, por otra parte, se ha relacionado con el cumplimiento de un criterio mediante la producción o el logro de un resultado en una tarea. El paradigma metodológico empleado para construir dichas tareas ha sido el procedimiento de igualación a la muestra, bajo el supuesto de que éste constituye una preparación experimental adecuada para estudiar fenómenos conductuales complejos, dada la facilidad de modificar el procedimiento, promoviendo así variaciones cuantitativas y cualitativas en el comportamiento de 
los individuos (Tena, Hickman, Moreno, Cepeda \& Larios, 2001).

En un procedimiento de igualación a la muestra típico se presentan arreglos de estímulos (ver Figura 1) donde algunos, usualmente dos, son llamados estímulos contextuales e indican un criterio de relación; otro es denominado estímulo muestra, y otros, generalmente tres, se conocen como estímulos de comparación (Ribes, 1990). Bajo estas condiciones, un individuo debe igualar uno de los estímulos de comparación con el de muestra, de acuerdo con el criterio relacional señalado por los estímulos contextuales. El procedimiento anteriormente descrito se conoce como igualación a la muestra de segundo orden (Tena et. al., 2001) ${ }^{2}$.

El referente conceptual sobre el cual descansan las interpretaciones de los hallazgos de esta línea de investigación proviene de la propuesta interconductual de J. R. Kantor (Kantor \& Smith, 1975), desarrollada posteriormente por E. Ribes (Ribes \& López, 1985). La metodología de investigación señalada ha servido, a la luz de esta propuesta teórica, para analizar las características morfológicas de la interacción psicológica implicada en ellas, tales como los elementos y funciones estimulativas de la tarea, y la adquisición y mantenimiento de las respuestas implicadas. También esta metodología ha sido útil para aproximarse a las características cuantitativas del comportamiento implicado en este tipo de situaciones experimentales, tales como las variaciones en los porcentajes de efectividad y en las latencias de respuesta. Por último, ha permitido estudiar las características cualitativas de la interacción, es decir, las maneras o modos funcionales en que ésta se da, teniendo como trasfondo los cinco niveles de organización del comportamiento propuestos por Ribes y López (1985).

Para estos autores, se entiende por nivel de organización del comportamiento la manera en que se establece una relación de interdependencia entre un individuo y su ambiente. La organización de dichos niveles está sustentada, por un lado, en la autonomía relativa de los sistemas reactivos con respecto a las propiedades fisicoquímicas y del aquí y el ahora, lo cual se conoce con el término de desligamiento (Ribes \& López, 1985). Por otra parte, dado que las relaciones entre un individuo y su medio que cualifican como psicológicas son las desligadas, se postula que el desligamiento es posibilitado por ciertos factores críticos o mediadores. Al papel que cumplen dichos factores se le conoce como mediación (Ribes, 1990).
Con base en el tipo de mediación y el criterio de inclusividad, los cinco niveles de organización del comportamiento propuestos por Ribes y López (1985) han sido agrupados de la siguiente manera. Los primeros tres niveles funcionales de la conducta, denominados contextual, suplementario y selector, se caracterizan por ser modos funcionales de interacción en los que existe aún dependencia del comportamiento con relación a las propiedades físicas y químicas de la situación inmediata y los parámetros espacio-temporales. Por esa razón, se dice que el desligamiento en estos casos es de tipo intrasituacional. El siguiente nivel funcional, llamado sustitutivo referencial, implica la participación de un sistema reactivo convencional que posibilita la interacción con eventos u objetos ausentes, que ocurren en diferente momento y lugar con relación a la situación inmediata. De aquí que se diga que este nivel de organización psicológica tiene una característica extrasituacional de desligamiento. Ribes y López (1985) aclaran, sin embargo, que en este nivel aún existe una dependencia con respecto a los eventos u objetos particulares con los que se interactúa, a pesar de que estén ausentes. El último nivel de organización de conducta, llamado sustitutivo no referencial, posee la característica transituacional de desligamiento, pues supone que las interacciones individuo-ambiente se dan al margen de cualquier propiedad física o biológica de los eventos u objetos de estímulo, y que son ajustes a relaciones entre productos exclusivamente convencionales. Por esta razón se dice que la interacción sustitutiva no referencial "trasciende" cualquier aspecto de la situación particular, caso ejemplificado en la formulación de leyes científicas y el razonamiento, entre otros productos convencionales del ser humano (Hurtado \& Muñoz, 2005; Ribes \& López, 1985).

Uno de los supuestos con los que se ha trabajado empíricamente es que las diferentes pruebas de transferencia permitirían evaluar cada uno de estos niveles de autonomía del comportamiento. La Tabla 1 presenta, de manera resumida, los tipos de prueba de transferencia correspondientes a cada nivel de interacción.

En la tradición de investigación que enmarca el presente trabajo, autores como Varela y Quintana (1995) han planteado una taxonomía de las pruebas de transferencia a partir de sus características en relación con el entrenamiento. Dichas características son: a) dimensión, que corresponde al límite que define el dominio de un criterio de efectividad y su funcionalidad. Ejemplos que enuncian los propios autores son las dimensiones de lo semántico, lo numérico y lo geométrico; b) relación: es el criterio compartido por dos o más objetos de estímulo; algunos ejem-

\footnotetext{
2 En este contexto de investigación es posible encontrar otras manipulaciones experimentales sobre diferentes parámetros (Flores, Ortega, Reyes, Mateos, Villanueva \& Amaya, 2005; Pérez-Acosta, Navarro \& Benjumea, 2002; Tena et. al., 2001).
} 
TABLa 1

CORRESPONDENCIA ENTRE NIVELES DE ORGANIZACIÓN DEL COMPORTAMIENTO, TIPO DE DESLIGAMIENTO Y TIPO DE PRUEBA USADA PARA SU EVALUACIÓN

$\begin{array}{lll}\text { Niveles de interacción } & \begin{array}{l}\text { Autonomía del comportamiento } \\ \text { en relación con la situación }\end{array} & \text { Tipos de prueba de transferencia }\end{array}$

Contextual

Suplementario Intrasituacional Intramodales

Selector

\begin{tabular}{lll}
\hline Sustitutivo referencial & Extrasituacional & Extramodales \\
\hline Sustitutivo no referencial & Transituacional & Extrarrelacionales \\
\hline
\end{tabular}

plos son las relaciones de semejanza y diferencia en la dimensión geométrica; o de sinonimia y antonimia en la dimensión semántica; c) modalidad: hace referencia a las propiedades de los objetos de estímulo, tales como forma, tamaño, color, entre otras; y, d) instancia: objeto de estímulo ante el cual responde un individuo.

Con base en esta clasificación, Varela y Quintana (1995) se aproximaron al problema de las manipulaciones dimensionales de las situaciones de prueba, campo que hasta ese momento no había sido explorado, y del cual sólo se conocen a la fecha dos estudios posteriores (Hurtado \& Muñoz, 2005; Pérez, 2002). Varela y Quintana estudiaron la transferencia extradimensional entre lo geométrico (GEO), lo semántico (SEM) y lo numérico (NUM), al exponer a niños de primaria y secundaria a diferentes órdenes de presentación de dimensiones: GEO-SEM-NUM; SEM-NUM-GEO; GEO-NUMSEM, y una condición en la que los participantes se exponían a una dimensión exclusivamente. A pesar de lograr la transferencia, se encontró un efecto de irreversibilidad en el comportamiento de quienes fueron entrenados inicialmente en la dimensión semántica. Por otra parte, la dimensión numérica fue la que ofreció mayor dificultad a los participantes, ya que tomó más tiempo para ser aprendida y dio lugar a pobres ejecuciones en transferencia.

Pérez (2002) reporta un estudio posterior en el que se diseñaron tareas de igualación manipulando las dimensiones geométrica, pictórica, semántica y numérica. Se tomó como criterio de igualación en entrenamiento y fase de prueba, tanto las propiedades físicas de los arreglos de estímulos, como sus propiedades convencionales. Este estudio contempló la distribución de 35 participantes en siete grupos experimentales; en cuatro de ellos se les entrenó para igualar en términos, de las propiedades físicas de los estímulos en una de las dimensiones (e. g., igualar dos dibujos con base en su forma y color). A pesar de que en la tradición de investigación descrita hasta el momento se ha hecho un uso extensivo de las pruebas de transferencia en tareas de igualación a la muestra, éstas parecen haber tenido únicamente un papel subsidiario a la hora de evaluar la interacción de variables ajenas a las pruebas en sí mismas. Gran parte de las características definitorias de las pruebas no han sido exploradas, pues en las investigaciones sus aspectos se mantienen constantes o no se reportan (e. g., tipo de prueba, orden de presentación, restricciones en el tiempo de respuesta, etcétera).

El presente estudio indagó qué ocurre con las ejecuciones de sujetos humanos en tareas de igualación de segundo orden, cuando se altera la manera tradicional en que se han presentando las pruebas de transferencia (nivel de dificultad ascendente) y se introducen además niveles superiores de dificultad/complejidad poco explorados, como es el caso de los cambios extradimensionales, ubicados en los últimos niveles de la Matriz de Transferencia Competencial (MTC) propuesta por Varela y Quintana (1995).

Se buscó, entonces, caracterizar y comparar las ejecuciones de los participantes ante cada una de las pruebas de transferencia típicamente utilizadas (intramodales y extramodales). Por otra parte, se exploraron los desempeños ante variaciones poco estudiadas hasta la fecha, como son las pruebas extrarrelacionales y extradimensionales.

Un primer paso para ello fue identificar las variaciones específicas de instancia, modalidad, relación y dimensión propias de cada prueba de transferencia, para establecer después su lugar en la MTC propuesta por Varela y Quintana (1995). De esta manera, se daban las condiciones para contrastar los resultados de este estudio con las predicciones de dificultad que se derivaban de dichos niveles. El objetivo fue avanzar hacia el refinamiento de la matriz, comprobando sus supuestos teóricos y mostrando la validez de las pruebas de transferencia 
para evaluar los diferentes niveles de autonomía del comportamiento (intrasituacional, extrasituacional y transituacional).

La pregunta general de investigación que guió el análisis de los resultados fue: ¿Cuál es el efecto de manipular el tipo y el orden de exposición a las pruebas de transferencia, sobre las ejecuciones de los participantes en una tarea de igualación a la muestra? De esta pregunta general pueden derivarse los siguientes interrogantes específicos, que buscó responder el presente estudio: ¿Qué orden de exposición a las pruebas de transferencia (ascendente, descendente o aleatorio) posibilita u obstaculiza un mayor ajuste conductual de los participantes? Teniendo en cuenta la ejecución de los participantes en cada una de las pruebas de transferencia, al margen de su orden de exposición: ¿Cómo se ordenan dichas pruebas en términos del ajuste de las ejecuciones de los participantes al criterio de efectividad?

\section{Método \\ Participantes}

Los participantes fueron veintiocho estudiantes universitarios de pregrado de diferentes carreras de la Facultad de Ciencias Humanas, de la Universidad Nacional de Colombia, con edades entre los 16 y los 29 años (16 mujeres y 9 hombres); en el caso de tres de ellos, esta información no apareció registrada. Fueron escogidos por conveniencia bajo el criterio de que ninguno de ellos tuviera experiencia previa con el tipo de tareas contempladas en este trabajo.

\section{Instrumento}

La aplicación del instrumento se llevó a cabo en las instalaciones de los laboratorios de Psicometría y Cognición, en las oficinas de Coordinación de Redes de la Facultad de Ciencias Humanas de la Universidad Nacional de Colombia, en un horario de 2 a 5 p.m. Se emplearon equipos de cómputo con monitores de 15" y velocidad de procesamiento superior a $700 \mathrm{mhz}$. La programación de la tarea asistida por computador se realizó utilizando el programa Macromedia-Authorware 6.0, bajo ambiente Windows.

\section{Procedimiento}

Una vez recogidos los datos de cada participante (nombre, edad y ocupación) por medio de un formulario en pantalla, uno de los investigadores le indicaba que podía empezar y le aclaraba que podría solucionarle inquietudes únicamente durante los momentos en que se mostrara las instrucciones y el ejemplo de la tarea en la pantalla.
Una vez hecho esto, aparecían las siguientes instrucciones (cada párrafo corresponde a lo que el participante veía en la pantalla; posteriormente debía pulsar el botón "Continuar" para pasar al siguiente):

(Nombre del participante): Queremos darte la bienvenida y agradecer tu participación en este estudio sobre aprendizaje. Por favor, con el "ratón" presiona el botón "continuar". (Continuar) El juego que diseñamos en este programa no es una prueba ni de inteligencia, ni de memoria, ni de personalidad sino que trata de explorar algunos procesos de solución de problemas que son comunes a la mayoría de las personas. (Continuar) Tus datos personales serán empleados para fines investigativos exclusivamente y para hacerte posteriormente entrega de tus resultados individuales. (Continuar). El juego consiste en lo siguiente. En la pantalla aparecerán seis figuras: dos arriba, una en el centro y tres abajo. Debes elegir una de las de abajo, aquella que creas que se relaciona con la del centro, usando el "ratón”. El tipo de relación que existe entre la figura del centro y la de abajo estará indicada por las dos figuras que aparecen arriba. (Continuar) $\mathrm{Al}$ comienzo se te informará si cada elección que haces es correcta o incorrecta. Pero más adelante no se te proporcionará esta información; por eso es importante que prestes atención desde el comienzo. (Continuar) $\mathrm{Al}$ iniciar puede ser que no sepas qué elección hacer, pero a medida que avance el juego aprenderás varias cosas. La idea es que acumules el mayor número de respuestas correctas posible. (Continuar) Para que te familiarices con los ensayos que componen el juego enseguida aparece un ejemplo... [A continuación aparecía un ejemplo en la pantalla, el cual, independientemente de la respuesta que diera el participante, indicaba que la elección había sido correcta]

A partir de este momento comenzaban las diferentes fases de la tarea experimental. Cada participante debía escoger con el "ratón" el estímulo de comparación que considerara correcto en cada uno de los arreglos de igualación a la muestra de segundo orden (ver Figura 1). Al finalizar cada ensayo, y dependiendo de la elección del participante en la fase de entrenamiento, aparecía en la pantalla, durante un segundo, un mensaje de "correcto" o "incorrecto". Una vez concluida la fase de entrenamiento, la retroalimentación se retiraba; durante los ensayos correspondientes a la fase de prueba, a los participantes no se les indicaba si sus respuestas habían sido correctas $\mathrm{o}$ incorrectas.

Terminada la fase de pruebas aparecía un mensaje de despedida y agradecimiento en la pantalla. Los datos del participante eran guardados automáticamente por el software en el disco duro del computador para ser luego 
Figura 1.

EJEMPLO DE LOS ARREGLOS DE ESTÍMULO UTILIZADOS DURANTE EL EXPERIMENTO

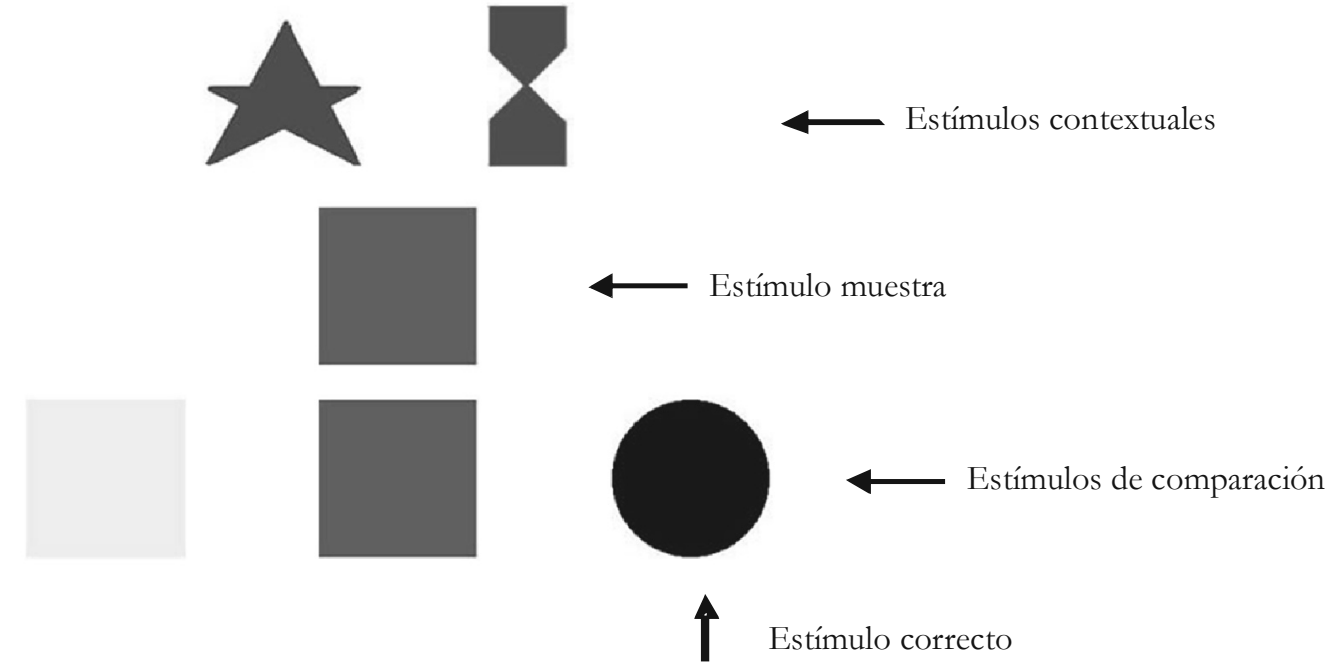

Nota. Elaborado con base en Psicología general, por E. Ribes, 1999, México: Trillas.

La opción correcta es el estímulo de la derecha (círculo azul), dado que la relación especificada por los estímulos contextuales es la de diferencia.

retirados por los experimentadores al final de las sesiones del día.

\section{Estímulos utilizados y condiciones de cada fase del experimento}

Entrenamiento. La fase de entrenamiento estuvo configurada para que los participantes respondieran entre tres y cuatro bloques, dependiendo del siguiente criterio: si la ejecución en los bloques 1, 2 y 3 era superior o igual al $90 \%$ de efectividad, los participantes no respondían el cuarto bloque y pasaban directamente a la fase de prueba. En caso contrario, debían pasar por los cuatro bloques de ensayos que conformaban la fase de entrenamiento antes de continuar con las pruebas.

Con el objetivo de garantizar una historia homogénea de preparación para todos los participantes, los bloques de entrenamiento tuvieron el mismo orden de presentación. Durante esta fase los estímulos de muestra y comparación fueron figuras geométricas (cuadrado, triángulo, rectángulo y círculo) con colores amarillo, verde, rojo y azul, mientras que los estímulos contextuales fueron figuras geométricas, diferentes a las muestras y comparaciones, en colores café y violeta.

Los criterios de igualación correspondientes al entrenamiento fueron los siguientes: se consideraba como correcta la respuesta consistente en elegir el estímulo idéntico a la muestra, cuando dicha relación era ejemplificada por los estímulos de segundo orden; o el diferente, cuando la relación de diferencia era ejemplificada por dichos estímulos.

Una vez los participantes habían respondido los bloques correspondientes a la fase de entrenamiento, aparecía en la pantalla la siguiente instrucción: "Las condiciones de la tarea son iguales, pero desde este momento no se te informará si tu elección ha sido correcta o incorrecta". En adelante, los participantes respondían a los arreglos correspondientes a la fase de prueba, ahora en ausencia de retroalimentación.

Fase de prueba. La fase de prueba estuvo constituida por cuatro bloques de ensayos para cada una de las pruebas de transferencia contempladas en este estudio: intramodal, extramodal, extrarrelacional y extradimensional. El orden de presentación de las pruebas varió para cada grupo experimental (ver Tabla 2). A continuación se describen las características de cada una de ellas:

Intramodal. En esta prueba se emplearon configuraciones novedosas que constituyeron los arreglos de estímulos en entrenamiento, es decir, que los de muestra y comparación fueron figuras geométricas (cuadrado, triángulo, rectángulo y círculo) con colores amarillo, verde, rojo y azul, mientras que los contextuales fueron figuras geométricas, diferentes a las muestras y comparaciones, en colores café y violeta; las relaciones vigentes fueron las de identidad y diferencia. 
Extramodal. Para esta prueba los estímulos contextuales siguieron siendo las mismas figuras geométricas del entrenamiento, sólo que ahora aparecían sin color de relleno y variaban en dos tamaños de aproximadamente $70 \%$ de diferencia. Así mismo, los estímulos muestra y comparación fueron las figuras geométricas y colores utilizados en el entrenamiento, los cuales también variaban en dos tamaños, con una diferencia aproximada del $70 \%$ de diferencia. En esta prueba se consideró correcta a la respuesta de elección del estímulo idéntico en forma, tamaño y color, o el diferente en forma y tamaño, según lo indicaran los estímulos de segundo orden. La modalidad color fue reemplazada por la modalidad tamaño, considerada relevante para la relación de diferencia.
Extrarrelacional. Se utilizó el mismo tipo de arreglos del entrenamiento, sin embargo, la relación de semejanza por forma o color reemplazó a la de diferencia como relación vigente. La relación de identidad conservó su vigencia durante esta prueba.

Extradimensional. Se cambiaron los arreglos geométricos por arreglos con palabras. Las relaciones vigentes fueron las de sinonimia y antonimia. En esta prueba se consideró correcto escoger las palabras sinónimas y antónimas, según lo señalaran los estímulos de segundo orden.

En todos los casos anteriores, responder a relaciones entre estímulos ajenas a las descritas se tomó como respuesta incorrecta. El lector podrá apreciar en la Figura

TABLA 2

DiSEÑO EXPERIMENTAL DEL ESTUDIO CON SUS CORRESPONDIENTES GRUPOS, FASES Y TAREAS CONSTTTUTIVAS

\begin{tabular}{llllll} 
Grupo & Entrenamiento & \multicolumn{2}{l}{ Secuencia de prueba } \\
\hline 1 & & A & B & C & D \\
& & TIM & TEM & TER & TED \\
2 & Relaciones & D & C & B & A \\
3 & deidentidad & TED & TER & TEM & TIM \\
& Concurrentemente & B & C & A & D \\
4 & & TEM & TER & TIM & TED \\
& & C & A & D & B \\
& & TER & TIM & TED & TEM \\
\hline
\end{tabular}

Nota TIM = Transferencia intramodal, TEM $=$ Transferencia extramodal, TER $=$ Transferencia extrarelacional, TED $=$ Transferencia extradimensional.

FIGURA 2

EJEMPLOS DE LOS ARREGLOS UTILIZADOS EN LAS DIFERENTES FASES DE LA TAREA EXPERIMENTAL

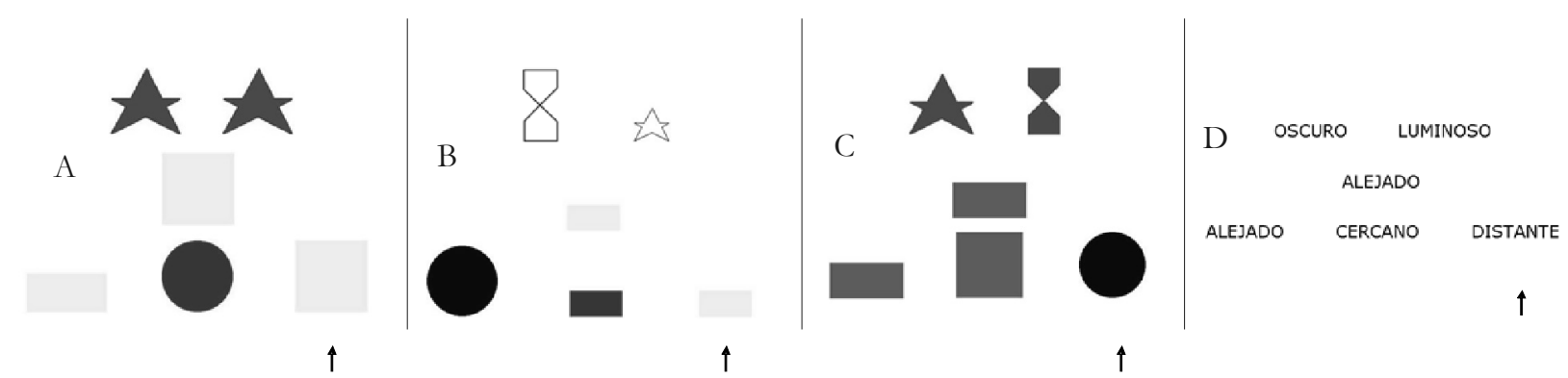

Nota. Las flechas indican la respuesta correcta en cada caso. El primer arreglo (A) corresponde al tipo de ensayo de las fases de entrenamiento y prueba intramodal. El segundo arreglo (B) corresponde al tipo de arreglo utilizado en la prueba extramodal. El arreglo que aparece de tercero $(\mathrm{C})$ corresponde a un ensayo típico de la prueba extrarrelacional, y, por último, el cuarto arreglo (D) corresponde a un ejemplo de los ensayos utilizados en la prueba extradimensional. 
2 un ejemplo de cada uno de los tipos de arreglo utilizados en este experimento.

Cada bloque de las fases de entrenamiento y prueba estuvo constituido por 24 ensayos y el número de éstos para cada relación, tanto en el entrenamiento como en las pruebas, fue balanceado. Estos ensayos eran presentados de forma concurrente, es decir, en un mismo bloque y de forma aleatoria aparecían ensayos correspondientes a dos relaciones. Un aspecto que fue contemplado para la construcción de los ensayos que requerían figuras geométricas como estímulos fue la cantidad de figuras y colores que aparecían en pantalla. En la mitad de todos los ensayos, los arreglos estuvieron constituidos por figuras que guardaban una configuración de dos colores y tres formas, mientras que en el resto de ellos se dio la configuración inversa: tres colores y dos formas.

La Tabla 2 presenta la distribución de los participantes en cada una de las cuatro condiciones experimentales.
Todos fueron expuestos al mismo tipo de entrenamiento, el cual consistía en una tarea concurrente de las relaciones de identidad y diferencia con estímulos geométricos.

Los grupos experimentales se diferenciaron según el orden de presentación de las pruebas de transferencia (intramodal, extramodal, extrarrelacional y extra-dimensional) al cual fueron expuestos. Los participantes respondían a una secuencia de cuatro bloques en los que, dependiendo del grupo al cual había sido asignado cada participante, el orden de aparición de las pruebas variaba. Para los grupos 3 y 4 el orden de aparición de las pruebas fue asignado al azar (ver Tabla 2). Es importante aclarar que el cambio entre cada bloque no era indicado por ninguna clave ni interrupción.

En términos de la matriz de transferencias posibles propuesta por Varela y Quintana (1995), la prueba intramodal usada en este estudio correspondería al caso más simple de variación de la situación de prueba con respecto al entrenamiento: prueba intradimensional-

FIGURA 3

EJECUCIÓN DE LOS PARTICIPANTES DURANTE LA FASE DE ENTRENAMIENTO EN TÉRMINOS DEL PORCENTAJE DE ACIERTO DURANTE LOS CUATRO BLOQUES.
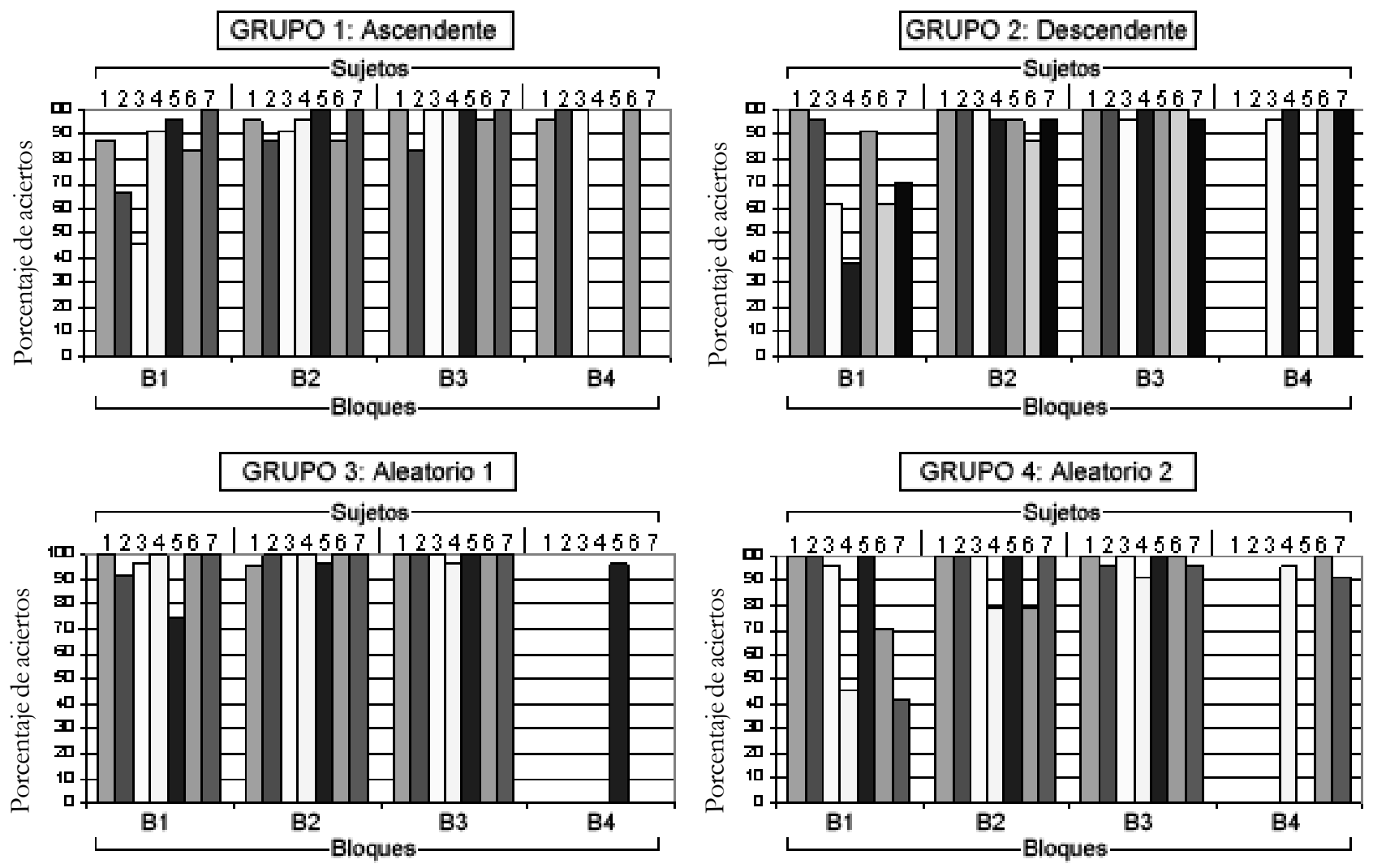

Nota. Cada barra representa uno de los siete participantes por grupo. B1 = bloque 1, B2= bloque 2, B3= bloque 3, B4= bloque 4. 
FIGURA 4

EJECUCIÓN DE LOS PARTICIPANTES DURANTE LA FASE DE PRUEBA EN TÉRMINOS DEL PORCENTAJE DE ACIERTOS EN CADA UNO DE LOS TIPOS DE TRANSFERENCIA
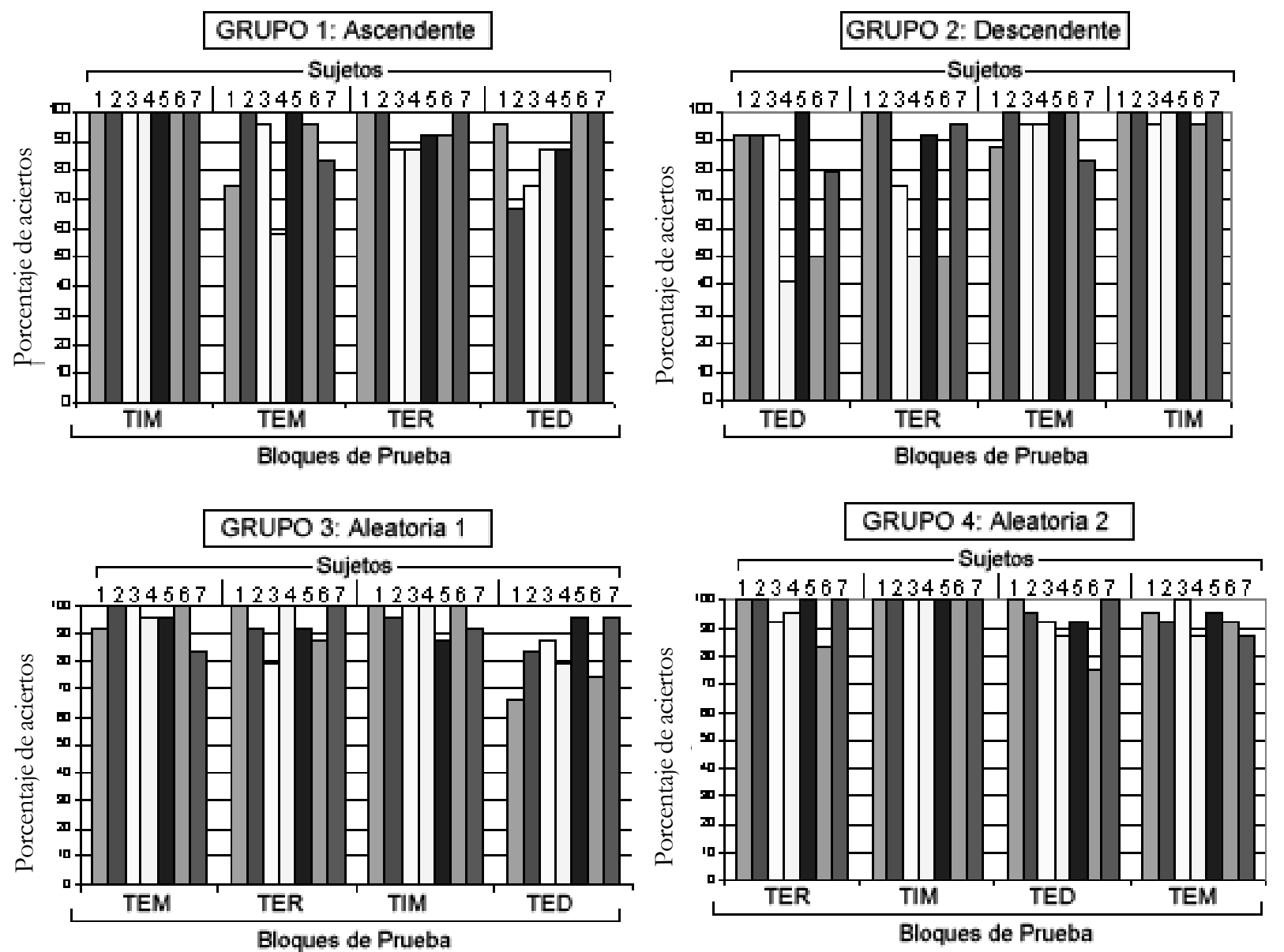

Nota. Cada barra representa uno de los siete participantes por grupo. TIM = Transferencia intramodal; TEM = Transferencia extramodal; TER = Transferencia extrarrelacional; TED = Transferencia extradimensional.

intrarrelacional-intramodal-extrainstancia. La extramodal equivaldría a una prueba intradimensional-intrarrelacional-intrainstancia-extramodal. La extrarrelacional, a una prueba de tipo intradimensional-intramodalintrainstancia-extrarrelacional. Por último, la prueba de transferencia extradimensional pertenecería a una prueba intrarrelacional-extrainstancia-extramodal-extradimensional.

\section{Resultados}

\section{Entrenamiento}

La Figura 3 muestra la ejecución de los participantes en cada uno de los grupos experimentales y en los diferentes bloques de ensayos durante la fase de entrenamiento.

En los grupos 1 y 2 se observa que, a partir del primer bloque, tres de los siete participantes ya lograban cumplir con el criterio de efectividad del $90 \%$ (ver Figura 3). En el grupo 3 esta tendencia es más acentuada, pues se observa que seis de los siete participantes cumplieron el criterio desde el primer bloque. En el caso del grupo 4, cuatro de los siete participantes tuvieron ejecuciones superiores al 90\% de efectividad durante el primer bloque. El grupo 3 presentó los porcentajes de efectividad más homogéneos en una menor cantidad de ensayos, ya que a la altura del bloque 2 todos sus integrantes ya habían cumplido el criterio del $90 \%$ de efectividad. Para el tercer bloque del entrenamiento, 27 de los 28 participantes de todos los grupos experimentales ya habían cumplido el criterio de efectividad del 90\% en alguno de los bloques de entrenamiento. Solamente cuatro participantes de los grupos 1 y 2 , uno del grupo 3 y tres del grupo 4 tuvieron que responder al cuarto y último bloque del entrenamiento antes de pasar a la fase de prueba, debido a que 
no habían cumplido con el requisito de tener ejecuciones iguales o superiores al 90\% de efectividad durante los tres primeros bloques.

\section{Pruebas de transferencia}

Descripción de las ejecuciones por grupos durante las pruebas de transferencia

A continuación se presentan los resultados de las ejecuciones de los participantes en cada uno de los grupos experimentales durante la fase de transferencia.

Como muestra la Figura 4, en el grupo 1 (orden ascendente: TIM-TEM-TER-TED), el número de participantes que cumplieron el criterio fue disminuyendo a medida que pasaban los bloques: todos cumplieron con el criterio de efectividad prescrito $(90 \%)$ para la prueba TIM. Posteriormente, en la prueba TEM, cuatro lo cumplieron; los tres restantes que no lograron cumplir con el criterio (P1, P4 y P7) tuvieron ejecuciones entre el 75\% y $83 \%$. En la prueba TER, cinco participantes cumplieron el criterio con ejecuciones entre un $88 \%$ y un $100 \%$ de efectividad, y los dos restantes (P3 y P4) tuvieron más del $85 \%$ de aciertos. Finalmente, en la prueba TED, tres participantes cumplieron el criterio de efectividad, dos de ellos (P6 y P7) con un 100\% de aciertos y P1 con 96\%; los cuatro restantes que no cumplieron con el criterio tuvieron ejecuciones entre el $67 \%$ y $88 \%$ de efectividad.

La tendencia de decremento en el número de participantes que cumplían el criterio en el grupo 1 se mostró de forma inversa en el grupo 2 (Figura 4, parte superior derecha), donde, con una secuencia descendente TEDTER-TEM-TIM, cuatro de siete cumplieron con el criterio en la prueba TED (P1, P2, P3 y P5) y TER (P1, P2, P5 y $\mathrm{P7}$ ), mientras que el resto estuvieron por debajo del $80 \%$. Más adelante, cinco participantes cumplieron el criterio en el bloque TEM, tres de ellos (P2, P5 y P6) con un $100 \%$ de aciertos, y dos (P3 y P4) con ejecuciones de $96 \%$. Los dos restantes estuvieron cerca de alcanzar el criterio con porcentajes superiores a $80 \%$. Finalmente, en la prueba TIM, todos cumplieron el criterio de efectividad (ejecuciones entre 95 y 100\%).

En el caso del grupo 3 (Figura 4, parte inferior izquierda), el cual tuvo una secuencia asignada de manera aleatoria (TEM-TER-TIM-TED), seis de los participantes presentaron una ejecución igual o superior al 90\% durante la prueba TEM; el único que no cumplió con el criterio (P7), tuvo un 83\% de aciertos. Posteriormente, en la prueba TER, cinco participantes cumplieron el criterio, tres de ellos (P1, P4 y P7) con un 100\% de aciertos; los dos restantes (P2 y P5) tuvieron porcentajes alrededor del 80\%. En la prueba TIM, a diferencia de los de- más grupos experimentales, seis de los siete participantes cumplieron el criterio, cuatro de ellos con un $100 \%$ de aciertos (P1, P3, P4 y P6). El único que no lo hizo (P5) estuvo también por encima del $85 \%$. Al igual que en el caso del grupo 1, en el grupo 3 los participantes se expusieron al final de la fase de prueba a un bloque de ensayos de tipo extradimensional (TED). De manera muy semejante a la tendencia en el grupo 1, solamente dos (P5 y P7) cumplieron con el criterio de efectividad del $90 \%$, y los restantes tuvieron porcentajes cercanos superiores al 80\% (P2 y P3), mientras que otros tuvieron ejecuciones aún más bajas.

El grupo 4, el cual también tuvo un orden de presentación de las pruebas asignado de forma aleatoria (TER-TIM-TED-TEM), en comparación con los demás grupos, mostró las ejecuciones más homogéneas y con un mayor número de participantes que cumplieron el criterio de efectividad a lo largo de todas las pruebas (ver Figura 4, parte inferior derecha). En la prueba TER, seis cumplieron el criterio de efectividad, cuatro de ellos con un 100\% (P1, P2, P5 y P7); el único que no logró el criterio (P6) tuvo un 83\% de aciertos. En la prueba TIM, todos cumplieron el criterio con una efectividad del 100\%. En la prueba TED, cinco cumplieron el criterio, dos de ellos con un 100\% de aciertos (P1y P7), mientras los otros tres tuvieron porcentajes de aciertos entre el $92 \%$ y $96 \%$; los dos que no cumplieron con el criterio de efectividad (P4 y P6) tuvieron ejecuciones de 88\% y 75\% respectivamente. En la prueba final (TEM), también cinco participantes cumplieron el criterio, pero sólo uno con un $100 \%$ de aciertos (P3); los dos que no cumplieron con el criterio de efectividad (P4 y P7) tuvieron, en ambos casos, un $88 \%$ de aciertos.

Un aspecto importante que se hace evidente cuando se comparan las ejecuciones de los participantes en las pruebas de transferencia, que siguieron inmediatamente a la fase de entrenamiento, es que las diferencias no son considerables entre las pruebas TIM, TEM y TER, a diferencia de TED: cuando la prueba TIM apareció inmediatamente después del entrenamiento (grupo 1) todos los participantes cumplieron el criterio de efectividad. En el caso de las pruebas TEM (grupo 3) y TER (grupo 4), seis de los siete integrantes de cada grupo cumplieron el criterio y los restantes tuvieron ejecuciones cercanas al criterio de efectividad. En cambio, cuando la prueba TED apareció inmediatamente después de la fase del entrenamiento (grupo 2), solamente cuatro de los siete miembros de este grupo cumplieron el criterio y el resto tuvieron ejecuciones lejanas al porcentaje de efectividad del 90\% (ver Figura 4). 
TABLA 3

NÚMERO DE PARTICIPANTES QUE CUMPLIERON CON EL CRITERIO DE EFECTIVIDAD SEGÚN CADA TIPO DE PRUEBA DE TRANSFERENCIA Y EN CADA UNO DE LOS GRUPOS EXPERIMENTALES

\begin{tabular}{llllll} 
Grupo & TIM & TEM & TER & TED & Totales \\
\hline 1 (ascendente) & 7 & 4 & 5 & 3 & 19 \\
2 (descendente) & 7 & 5 & 4 & 4 & 20 \\
3 (aleatorio) & 6 & 6 & 5 & 2 & 19 \\
4 (aleatorio) & 7 & 5 & 6 & 5 & 23 \\
Totales & 27 & 20 & 20 & 14 & \\
\hline
\end{tabular}

Descripción de las ejecuciones de todos los participantes en cada tipo de prueba

En la Tabla 3 se presenta el total de participantes que cumplieron el criterio de efectividad del $90 \%$ en cada uno de los tipos de prueba, al margen de su posición en las secuencias de cada grupo. Los datos del estudio muestran que la prueba con mayor número de participantes con ejecuciones efectivas es la TIM con 27/28 participantes, seguida de las pruebas TEM y TER, con 20/28 participantes respectivamente. Cabe resaltar que para Varela y Quintana (1995), estas tres pruebas son de tipo intradimensional (pertenecen, en este caso, a la dimensión geométrica). Finalmente, en el caso de la prueba TED, sólo 14 participantes de 28 alcanzaron el criterio de efectividad prescrito. El orden en que se agruparon las pruebas corresponde a la jerarquía de dificultad para los tipos de transferencia propuesta por Varela y Quintana (1995).

\section{Discusión}

Patrones de adquisición durante la fase de entrenamiento y su relación con las ejecuciones durante la fase de prueba

Si bien el diseño experimental suponía una historia de entrenamiento homogénea para todos los participantes de este estudio, los resultados mostraron diferencias considerables en cada uno de los grupos en cuanto a velocidad de adquisición de las discriminaciones condicionales (ver Figura 3) y la cantidad de bloques que requirieron los diferentes sujetos para cumplir con el criterio de efectividad para pasar a la fase de prueba. Este aspecto, además, pareció interactuar con las características de las pruebas que siguieron al entrenamiento, lo que apoya así la interpretación que sugiere que una menor exposición a tareas que exigen un ajuste basado en las circunstancias inmediatas favorece posteriormente unas ejecuciones efectivas en ta- reas de mayor dificultad como TER y TED. En contraste, una mayor cantidad de bloques de exposición continua a tareas con énfasis en las contingencias inmediatas tienden a restringir el comportamiento a la situación original de adquisición.

Orden de exposición en las pruebas de transferencia $y$ ajuste conductual de los participantes

Los grupos con participantes que tuvieron ejecuciones más heterogéneas en el presente estudio fueron 1, 2 y 3 . Específicamente, los integrantes de los grupos 1 y 3 tuvieron las ejecuciones más bajas en la prueba TED, mientras que los del grupo 2 las tuvieron en las pruebas TED y'TER.

El aspecto compartido por los grupos 1 y 3 fue el haber iniciado la fase de transferencia con dos de las pruebas de menor dificultad (TIM y TEM), las cuales presentaban solamente variaciones de tipo instancia y modalidad. A pesar de que los participantes de estos grupos empezaron su fase de transferencia con buenas ejecuciones en las pruebas TIM y TEM, tuvieron pobres ejecuciones en momentos posteriores, cuando se enfrentaron a las pruebas de mayor dificultad (TER y TED). A partir de este patrón de ejecución se podría plantear que los integrantes de ambos grupos tuvieron una historia que "fijó" su comportamiento a los aspectos situacionales de la tarea. Luego de exponerse a tres o cuatro bloques de entrenamiento, los sujetos se enfrentaron a cambios durante la fase de prueba que no configuraron una situación demasiado diferente a la de entrenamiento (cambios en modalidad e instancia). Así, las pruebas TIM y TEM fueron dos bloques de ensayos adicionales con características muy similares a las del entrenamiento, y el criterio de ajuste también estuvo centrado todo el tiempo en las contingencias inmediatas. Sin embargo, cuando estos mismos participantes se expusieron a cambios 
que exigieron ajustes superiores al nivel intrasituacional (propios de las pruebas TER y TED), las ejecuciones efectivas y autónomas, con respecto a las características de los arreglos de estímulo y las contingencias vigentes en ese momento, se hicieron menos probables $^{3}$.

El caso del grupo 2 apoya la anterior interpretación, pues aquellos participantes que tuvieron una menor cantidad de bloques de entrenamiento, porque cumplieron con el criterio que evitaba que se expusieran a un cuarto bloque, fueron precisamente los que lograron mejores ejecuciones en la fase de prueba TED, que apareció inmediatamente después del entrenamiento. Además, durante el resto de pruebas subsiguientes dicha efectividad se mantuvo, a pesar de los cambios intra o extrasituacionales posteriores. De esta manera, los resultados parecen sugerir que un número menor de bloques de entrenamiento evita que el comportamiento quede "fijado" a aspectos situacionales, que de otra manera obstaculizarían una ejecución alta en pruebas con cambios tan pronunciados como los de las extradimensionales. Sin embargo, también podría interpretarse que la velocidad de aprendizaje es un predictor confiable de buenas ejecuciones en este tipo de pruebas. Eso querría decir que los sujetos que aprendieron rápidamente se comportaron también más inteligentemente, en el sentido en que fueron efectivos en las pruebas y de forma más variada.

Por otra parte, en el grupo 4, cuyo orden de presentación de las pruebas fue establecido al azar, fue en el que más participantes se ajustaron al criterio de efectividad. Es necesario señalar que, después de pasar por al menos tres bloques de entrenamiento (en las relacio- nes de identidad y diferencia), estos participantes se enfrentaron a la prueba TER (conformada por las relaciones de identidad y semejanza), para seguir posteriormente con la TIM (conformada por las relaciones de identidad y diferencia).

Considerando únicamente la relación de identidad, los participantes del grupo 4 estuvieron expuestos a una fase de entrenamiento y dos bloques de prueba con las mismas condiciones situacionales, por lo tanto demandaban el mismo criterio de efectividad. Esto pudo conducir a que el cambio situacional implicado en la prueba TER se viera atenuado por el orden particular de las pruebas de este grupo, lo que propició que las ejecuciones en el segmento de pruebas TER-TIM resultara no sólo alto, sino también homogéneo. La explicación tradicional que puede darse a los resultados en estas dos pruebas es que el comportamiento de los participantes quedó, en algún momento de la tarea, bajo el control relacional explicitado en los estímulos de segundo orden. Este fenómeno se conoce en la literatura experimental como control abstracto del estímulo (Pérez, 2002). Es posible pensar en este tipo de control y las condiciones propiciadas por la secuencia TER-TIM como factores que, en conjunto, habrían llevado el comportamiento a los niveles de efectividad observados en estas pruebas (ver Figura 4).

Los cambios situacionales drásticos para el grupo 4 aparecieron con la prueba TED (tercera en la secuencia), en la cual, a pesar de haber decrecido el número de participantes que venían cumpliendo con el criterio, el grupo obtuvo las mejores ejecuciones en TED encontradas en

\footnotetext{
3 A partir de una revisión del procedimiento de igualación a la muestra y su correspondencia con los elementos conceptuales definitorios de la conducta sustitutiva, Peña-Correal (2006, comunicación personal) ha señalado algunas dudas con respecto a las supuestas intrasituacionalidad y extrasituacionalidad evaluadas en las diferentes pruebas de transferencia. El siguiente es su argumento. La aproximación metodológica tradicional contempla el entrenamiento de discriminaciones condicionales en las que las relaciones entre estímulos se basan en propiedades formales (e.g., una o más modalidades relevantes: color, forma, tamaño, textura, etcétera). Luego de que los sujetos han alcanzado un criterio de efectividad en la fase de entrenamiento de dichas discriminaciones condicionales, se introducen una serie de variaciones en los arreglos de estímulo, ya sea a nivel de las instancias, modalidades o relaciones vigentes, y todas ellas en ausencia de retroalimentación (i.e. pruebas de transferencia). Se ha supuesto que precisamente las variaciones en las modalidades de los estímulos, denominadas pruebas extramodales, constituirían cambios que exigirían un desligamiento del comportamiento, al menos de tipo extrasituacional. De este argumento se deriva que dichas pruebas constituirían formas de evaluar la conducta del tipo sustitutivo referencial (Tena et al., 2001). Sin embargo, si se contempla que uno de los elementos conceptuales definitorios de la aptitud sustitutiva referencial es la transformación de las contingencias en virtud de la convencionalidad implicada en los estímulos y respuestas, la cual permite que los individuos respondan a objetos y eventos no presentes y/o a propiedades no aparentes, es evidente que las pruebas extramodales no garantizan dicho criterio conceptual, más aún cuando tradicionalmente las variaciones en las modalidades son en una dimensión física (e. g., cambiar la modalidad relevante "color" por "tamaño") y las propiedades a las cuales continúa respondiendo el individuo durante la prueba extramodal son aparentes. Inclusive, Hurtado (2006) ha sugerido que, para responder efectivamente una prueba de tipo extramodal, hipotéticamente no se requeriría nada más que una historia extensa de efectividad durante el entrenamiento con figuras geométricas y propiedades y relaciones aparentes de las mismas, y en cambio no serían críticos otros requisitos de la función sustitutiva referencial, por ejemplo un repertorio convencional. Según Hurtado, aunque una prueba empírica de la anterior hipótesis contemplaría un estudio experimental (hasta la fecha no realizado) en el que se sometiera a individuos que no posean repertorios convencionales de un entrenamiento similar y un conjunto de pruebas de transferencia de tipo extramodal, los planteamientos anteriores dejan sospechas acerca de la viabilidad de este tipo de situaciones experimentales para estudiar los fenómenos sustitutivos referenciales.
} 
este estudio (cinco cumplieron el criterio del $90 \%$, y uno de los dos restantes un $88 \%$ de efectividad). Este alto nivel de ejecución puede tener explicación en el ya mencionado fenómeno del establecimiento de control contextual por parte de los estímulos de segundo orden. A pesar de estar expuestos al cambio más radical (TED), los participantes del grupo 4 siguieron ajustando su comportamiento de acuerdo con el criterio relacional impuesto por dichos estímulos, ya no con base en las propiedades físicas de éstos, sino con base en las propiedades arbitrarias/convencionales propias de la dimensión semántica. Esto último puede tener lugar siy sólo si los participantes comparten la convención patente en la tarea extradimensional ${ }^{4}$. Una completa fijación a las características situacionales/ físicas de la tarea y la ausencia de control contextual por parte de los estímulos de segundo orden se habría reflejado en una pobre ejecución en TED, lo cual no ocurrió, a la luz de los resultados obtenidos en este grupo.

Las altas ejecuciones encontradas en TEM (ver Figura 4), prueba con la que culminaba la fase de transferencia del grupo 4, confirman la hipótesis del desarrollo de control abstracto del estímulo, el cual pudo verse refinado a lo largo de las sesiones de prueba, tal como éstas quedaron dispuestas para este grupo.

\section{Análisis del ordenamiento de las pruebas de trans- ferencia en función de la ejecución de los partici- pantes ante cada una de ellas}

Tener cada una de las pruebas de transferencia inmediatamente después de la fase de entrenamiento brindó la oportunidad de observar la ejecución típica de los participantes ante cada una de ellas, dada además una historia homogénea de adquisición, y en ausencia de cualquier efecto proveniente de la configuración del conjunto de pruebas. De esta manera, fue posible observar el ordenamiento de las pruebas en función del ajuste diferencial que presentaron las ejecuciones de los participantes.

En consecuencia, TIM se encontraría en primer lugar en dicho ordenamiento, puesto que las siete personas que se expusieron a esta prueba después del entrenamiento cumplieron con el criterio de efectividad. En este ordenamiento seguirían TEM y TER; para ambos casos, seis de los siete participantes lograron el criterio cuando estas pruebas siguieron al entrenamiento. Es importante señalar que en ambas pruebas aquellos participantes que no cumplieron el criterio de efectividad del $90 \%$ tuvieron altas ejecuciones (superiores al 80\%). La prueba que ocupa el último lugar en esta clasificación es TED, ante la cual solamente cuatro participantes cumplieron el criterio de efectividad cuando pasaron inmediatamente del entrenamiento a ella. En contraste con el caso de las pruebas TEM y TER, los tres participantes que no lograron el criterio de efectividad de la prueba TED tuvieron ejecuciones inferiores al $80 \%$ de efectividad, aspecto que ratifica este ordenamiento.

Esta clasificación de las pruebas permite determinar su relevancia para poder evaluar el grado de autonomía del comportamiento desplegado ante cada una de ellas, dada una historia previa de entrenamiento que determina las características situacionales. Podría, entonces, compararse el patrón de ordenamiento encontrado en este estudio con la propuesta conceptualizada tradicionalmente (Tena et al., 2001). Son precisamente estas consideraciones las que serán tratadas a continuación con mayor detalle.

\section{Una propuesta de trabajo para evaluar la relación entre niveles de desligamiento y pruebas de trans- ferencia}

Tradicionalmente, en los estudios encaminados a la investigación de procesos sustitutivos, se ha considerado que las pruebas intramodales (TIM) evalúan características intrasituacionales del comportamiento; las extramodales (TEM), características extrasituacionales; y las extrarrelacionales (TER), características transituacionales (Tena et al., 2001). Por su parte, el papel de las pruebas extradimensionales no ha sido considerado a propósito de la correspondencia entre éstas y los niveles de dependencia situacional del comportamiento. Inclusive, tal como se mencionaba en la parte introductoria de este estudio, han sido pocas las investigaciones dedicadas a explorar este tipo de variaciones.

En esta misma tradición ha existido la tendencia a asumir implícita o explícitamente la dificultad de una prueba de transferencia como un indicador de complejidad del comportamiento desplegado. Dicha tendencia se puede ver ejemplificada en las predicciones que Varela y Quintana (1995) hacen con respecto a las ejecuciones efectivas de los participantes frente a cada uno de los 15

\footnotetext{
4 Debe aclararse que este asunto no fue controlado explícitamente en este estudio. En cambio, se asumió que todos los sujetos compartían dicha convención dadas las características de los estímulos lingüísticos utilizados (palabras sencillas y de amplio uso cotidiano). Estudios posteriores, como el de Hurtado y Muñoz (2005), demuestran cómo este factor debe controlarse con mayor detalle. Por ejemplo, estos autores utilizaron un cuestionario de sinónimos y antónimos para garantizar que todos los sujetos “jugaban” bajo el mismo sistema convencional. Así, las diferencias en las ejecuciones ya no podrían atribuírsele a las propiedades mismas de los estímulos utilizados.
} 
TABLA 4

RELACIÓN ENTRE EL PROMEDIO DE LATENCIA ENCONTRADO EN CADA PRUEBA Y EL NÚMERO DE PARTICIPANTES QUE CUMPLIÓ EL CRITERIO DE EFECTIVIDAD EN CADA UNA DE ELLAS

\begin{tabular}{llcl} 
Tiempo(s) & Relaciones & $\begin{array}{c}\text { Efectividad (número de } \\
\text { participantes) }\end{array}$ & Nivel situacional \\
\hline $2-3 \mathrm{~s}$ & Identidad (TIM, TEMy TER) & $28 / 28$ & Intrasituacional \\
$4 \mathrm{~s}$ & Diferencia (TIM) & $26 / 28$ & Intrasituacional \\
$4-5 \mathrm{~s}$ & Semejanza (TER) & $16 / 28$ & Extrasituacional \\
$8-9 \mathrm{~s}$ & Diferencia (TEM) & $17 / 28$ & Extrasituacional \\
& Sinonimia (TED) & $13 / 28$ & Transituacional \\
& Antonimia (TED) & $22 / 28$ & Transituacional \\
\hline
\end{tabular}

tipos de prueba propuestos. Ésta es la manera como los autores identifican las diferencias en la dificultad de una prueba y el comportamiento desplegado durante ella.

Es en el contexto de esta tradición conceptual y de investigación que se discuten los hallazgos del presente estudio. Se explora cómo éstos coinciden o no con la correspondencia tradicionalmente asumida entre los niveles de dependencia situacional del comportamiento y el tipo de pruebas utilizadas para su evaluación, y el problema de la dificultad de éstas como indicador de complejidad del comportamiento exhibido durante su ejecución. A partir de este análisis se propone una correspondencia alternativa entre niveles funcionales de comportamiento y los tipos de pruebas apropiados para su evaluación.

Los resultados de este estudio indican que las diferencias en las ejecuciones de los participantes ante las pruebas TIM, TEM y TER, frente a las observadas durante la prueba TED, son muy pocas. Hallazgos similares se observaron en estudios previos en los que fueron manipuladas sólo dos de estas pruebas (TIM y TEM). En estos casos, los investigadores tampoco encontraron diferencias en las ejecuciones de los participantes, a pesar de que también habían tenido una historia de entrenamiento homogénea (Ribes, Torres \& Ramírez, 1996).

Específicamente, los datos del presente estudio no mostraron diferencias en las ejecuciones efectivas de los participantes frente a TEM y TER, hecho que permitiría suponer que ambas pruebas podrían ser pertinentes para evaluar niveles de desligamiento extrasituacionales. Sin embargo, esta aparente homogeneidad desaparece si se evalúa a la luz de un criterio diferente de dificultad de la tarea, consistente en los tiempos de latencia empleados por los participantes en cada una de las pruebas.

El ordenamiento de las pruebas de acuerdo con el incremento en los tiempos de latencia de respuesta fue el siguiente: TIM-TER-TEM-TED, patrón en el que se observa una coincidencia en la posición de las pruebas extremas de menor y mayor dificultad en la jerarquía propuesta por Varela y Quintana (1995) (TIM y TED), pero mostrándose invertidas precisamente las pruebas intermedias (TEM y TER). De darse una relación funcional entre las variables "latencia de respuesta" y "porcentaje de efectividad", una posible línea de análisis apuntaría a que un incremento en la primera (tiempos de latencia) debería verse acompañado de ejecuciones menos efectivas (porcentajes de efectividad bajos). Esto implicaría, a su vez, que menores tiempos de latencia deberían acompañar la mayor proporción de ejecuciones efectivas. En términos generales, se estaría afirmando que ambas variables constituyen indicadores de dificultad de las pruebas y de la complejidad del comportamiento.

Atendiendo a la Tabla 4, que muestra la relación entre el promedio de latencia encontrado en cada prueba y el número de participantes que cumplieron el criterio de efectividad en cada una de ellas, la última parte de la predicción -con respecto a la dificultad de la tarea, tiempos de latencia y efectividad-se confirma, ya que en la relación de identidad en TIM, TEM y TER, y la de diferencia en TER, sí se observa mayor efectividad acompañada de menores tiempos de latencia de respuesta. Sin embargo, el haber encontrado altas latencias acompañadas de altas ejecuciones efectivas, como fue el caso para la relación de diferencia en TEM y antonimia en TED, lleva a reconsiderar la validez de los tiempos de latencia como una variable que siempre indica la dificultad de las pruebas y la complejidad del comportamiento desplegado en ellas.

De acuerdo con lo anterior, es posible sugerir que los tiempos de latencia cumplen un papel efectivo como indicadores de dificultad de una prueba (y posiblemente de complejidad del comportamiento) cuando ésta exige a 
TABLA 5

Propuesta DE REAGRUPACIÓN DE LOS TIPOS DE PRUEBAS DE TRANSFERENCIA Y SU CORRESPONDENCIA CON LOS NIVELES DE AUTONOMÍA CON RESPECTO A LO SITUACIONAL

Tipos de prueba de transferencia

Autonomía del comportamiento en

relación con lo situacional

\begin{tabular}{ll}
\hline Intramodal & Intrasituacional \\
Extramodal & \\
\hline Extrarrelacional & Extrasituacional \\
Extradimensional & Transituacional \\
\hline
\end{tabular}

los individuos ajustarse únicamente a las propiedades intrasituacionales, pero tal papel conjunto deja de ser claro a la hora de enfrentar a los participantes a las propiedades extra o transituacionales. En tales casos, es posible que la latencia siga mostrando la dificultad inherente a los arreglos de estímulo en su dimensión perceptual, pero la respuesta efectiva dependerá en mayor parte de factores vinculados a la complejidad misma de la interacción exigida (tales como el nivel de desligamiento y el tipo de mediación implicados). En el caso de este estudio, es posible que los participantes "supieran" cuál estímulo debían escoger, y la demora podría radicar en el tiempo que tomaría detectar las características que permitían discriminar el estímulo correcto. Tal interpretación, de confirmarse, podría dar cuenta de los elevados tiempos de latencia acompañados de una alta efectividad.

A manera de síntesis, en relación con la pertinencia de TEM y TER para evaluar la autonomía extrasituacional del comportamiento, es posible afirmar que se trata de un tema acerca del cual no puede darse una conclusión definitiva, con base en los resultados del presente estudio. Si bien el número de participantes que cumplieron con el criterio de efectividad en ambas pruebas es homogéneo, no ocurre lo mismo al atender a los promedios de tiempo de latencia de respuesta registrados para cada una de las relaciones constituyentes de dichas pruebas.

Todos lo elementos anteriormente considerados abren la posibilidad de asumir, a manera de hipótesis de trabajo para futuros estudios, que los cambios propios de las pruebas intramodales y extramodales parecen ser más válidos para evaluar aspectos intrasituacionales del comportamiento, mientras que los cambios extrarrelacionales corresponderían mejor a la evaluación de características extrasituacionales. De este modo se conservan las variaciones típicas de las pruebas extradimensionales como aspectos evaluativos de autonomía transituacional, al menos en cambios de la dimensión geométrica a la semántica, tal como ocurrió en este estudio. La Tabla 5 resume estas consideraciones alrededor de las pruebas y su relación con la autonomía del comportamiento en lo relativo a lo situacional.

Una propuesta alternativa de correspondencia entre los niveles de autonomía situacional del comportamiento y las pruebas útiles para evaluarlos, con la inclusión de las pruebas extradimensionales en tanto apropiadas para evaluar los niveles de autonomía transituacional, necesita de mayor apoyo empírico para fortalecer su viabilidad, más aún cuando el estudio presenta una limitación en el análisis de los datos con respecto a la prueba TER. Una característica de dicha prueba es que solamente el 50\% de los ensayos constituyeron legítimamente situaciones en las que se evaluó una relación no entrenada (la prueba estuvo constituida por 12 ensayos de identidad - relación entrenada originalmente - ensayos intramodales y 12 ensayos de semejanza - relación no entrenada - ensayos extrarelacionales). Dado esto, fue necesario analizar las ejecuciones de todos los sujetos del experimento en cada uno de los tipos de ensayos que constituyeron esta prueba, intentando verificar si la efectividad variaba cuando se contemplaba el bloque completo (24 ensayos mixtos) o cuando solamente se analizaba la mitad (12 ensayos extrarelacionales). Dado que el número de ensayos a verificar para cada bloque de prueba extrarrelacional era de 12 , cuando se consideró como criterio de ajuste $10 / 12$ ensayos correctos (83.3\%), la distribución de sujetos que cumplieron el criterio de efectividad fue exactamente igual a la que se muestra en la Tabla 3. Cuando se consideró un criterio de efectividad de 11/12 ensayos correctos (91.7\%), el número de sujetos que cumplió con el criterio en la prueba extrarrelacional cambió a un total de 16 (grupo 1: 4 sujetos; grupo 2: 3 sujetos; grupo 3: 4 sujetos; grupo 4: 5 sujetos). En el presente estudio se decidió analizar los datos teniendo como criterio 10 de 12 ensayos. Sin embargo, es importante señalar que 
futuras investigaciones (e. g., Pardo, 2005) deben corregir las características de esta prueba para evitar su inconsistencia con las demás

Por otro lado, propuestas como la presentada en este estudio abren la puerta a una revisión de la validez misma de las pruebas de transferencia. Al respecto, la evidencia preliminar apunta a que posiblemente éstas no solamente evalúan la generalidad (transferencia) de lo aprendido, sino que, de hecho, afectan dicha generalidad.

Otro interés investigativo que surge a partir de este trabajo consiste en poder determinar las posibles razones que llevaron a que unas condiciones homogéneas de entrenamiento derivaran en desempeños heterogéneos por parte de los participantes. Aquí parece indicarse que un factor como la extensión del entrenamiento es relevante al momento de enfrentarse a las pruebas.

Por último, se exploró la posibilidad de acudir a los tiempos de latencia de respuesta como una medida paralela sensible a la dificultad de una tarea de igualación. Sin embargo debe analizarse sistemáticamente su precisión cuando se evalúan ajustes situacionales en contraste con ajustes extra y transituacionales del comportamiento.

\section{Referencias}

Flores, C., Ortega, D., Reyes, K, Mateos, R., Villanueva, S. \& Amaya, A. (2005). Reversiones parciales y totales muestra-comparativo en igualación a la muestra con consecuencias diferenciales y no diferenciales. Universitas Pyshcologica, 4 (1), 43-57.

Hurtado-Parrado, H. C. (2006). Diseño y evaluación de una alternativa metodológica para el estudio del comportamiento sustitutivo referencial: el procedimiento de evaluación relacional (Hayes, Barnes-Holmes y Roche, 2001). Proyecto de Tesis de Maestría. Universidad Nacional de Colombia. Bogotá. Manuscrito no publicado.

Hurtado-Parrado, H. C., \& Muñoz, M. I. (2005). Efectos en la ejecución durante una prueba de transferencia extradimensional debidos a la presencia o ausencia de entrenamiento previo. Universidad Nacional de Colombia, Bogotá. Manuscrito no publicado.
Kantor, J. R. \& Smith N. W. (1975). The Science of Psychology: An Interbehavioral Survey. Chicago: Principia Press.

Martínez, H. (2001). Estudios sobre transferencia en comportamiento humano. En G. Mares \& Y. Guevara (Coord.), Psicología interconductual: avances en la investigación básica (pp. 37-58). México: UNAM FES Iztacala.

Pardo, N. C. (2005). Evaluación de la dificultad de diferentes pruebas de transferencia y del efecto de la variación del orden de presentación de las mismas en una tarea de igualación a la muestra de segundo orden. Tesis de grado profesional no publicada, Universidad Nacional de Colombia, Bogotá.

Pérez, R. (2002). Interacción entre la convencionalidad y el tipo de dimensión en un paradigma de igualación a la muestra. Tesis de grado profesional no publicada, Universidad Nacional de Colombia, Bogotá.

Pérez-Acosta, A., Navarro, J. I. \& Benjumea, S. (2002). Entrenamiento y transferencia de la autodiscriminación condicional en autistas. Universitas Psychologica, 1 (1), 40-51.

Ribes, E., (1990). Psicología general. México: Trillas.

Ribes, E. \& López, F. (1985). Teoría de la conducta: un análisis de campo y paramétrico. México: Trillas.

Ribes, E., Torres, C., \& Ramírez, L. (1996). Efecto de los modos de descripción en la adquisición y transferencia de una discriminación condicional de segundo orden en humanos adultos. Acta Comportamentalia, 4, 159-179.

Tena, O., Hickman, H., Moreno, D., Cepeda M., L. \& Larios, R., M. (2001). Estudios sobre comportamiento complejo. En G. Mares \& Y. Guevara, (Coord.), Psicología interconductual: avances en la investigación básica (pp. 59-110). México: UNAM FES Iztacala.

Varela, J. \& Quintana, C. (1995). Comportamiento inteligente y su transferencia. Revista Mexicana de Análisis de la Conducta, 21 (1), 47-66.

Varela, J., \& Ribes, E. (2002). Aprendizaje, inteligencia y educación. En E. Ribes (Ed.), Psicología del aprendizaje (pp.191-209). México: Manual Moderno. 